\title{
Research Progress on Femtosecond Laser Drilling of Cooling Holes in Aeroengines
}

\author{
Xueqin. $\mathrm{HOU}^{1, \text { a }}$, Zheng. ZHANG ${ }^{2}$, Xinling. LIU ${ }^{3}$ and Yong. YANG ${ }^{4}$ \\ ${ }^{1}$ Beijing Institute of Aeronautical Materials , AVIC Failure Analysis Center , Beijing 100095,China \\ School of Material Science and Engineering,Beihang University,Beijing 100083, China \\ Beijing Key Laboratory of Aeronautical Materials Testing and Evaluation, Beijing 100095,China \\ ${ }^{2}$ School of Material Science and Engineering,Beihang University,Beijing 100083, China \\ ${ }^{3}$ Beijing Institute of Aeronautical Materials, AVIC Failure Analysis Center , Beijing 100095,China \\ Beijing Key Laboratory of Aeronautical Materials Testing and Evaluation, Beijing 100095,China \\ ${ }^{4}$ State Key Lab of Transient Optics and Photonics,Xi'an Institute of Optics \& Precision \\ Mechanics,CAS,Xi'an 710068,China \\ aroky2000k@163.com
}

\begin{abstract}
Keywords: Femtosecond laser; drilling;cooling hole; aeroengine; research state; challenge
Abstract. Femtosecond laser can be used in fine processing of any material, especially for micro-nano precision machining. Cooling holes is the important technology used to improve performance of the aeroengines hot-end components such as turbine vanes and blades and is widely used in advanced aeroengines. With large quantity, small dimension and high quality requirement, cooling holes of aeroengines urgently need to develop advanced processing technology. In this paper, we first introduce advantages and research state of the femtosecond laser processing micro-holes in aeroengines. Subsequently, we discuss problems and challenges of femtosecond laser processing cooling holes in aeroengines. At last, we present that the key research areas of femtosecond laser processing cooling holes in aeroengines are as follows: interaction mechanisms between femtosecond laser and aeronautical materials should be fully explained, and system controlling methods of femtosecond laser processing should be established.
\end{abstract}

\section{Introduction}

The inlet temperature of aeroengine quickly increases as thrust-weight ratio of modern aeroengine becomes more and more greater. The inlet temperature of aeroengine whose thrust-weight ratio is 10 reaches to $1580^{\circ} \mathrm{C} \sim 1680^{\circ} \mathrm{C}$. When the ratio is $15 \sim 20$, the inlet temperature of aero-engine reaches to $2227^{\circ} \mathrm{C} \sim 2470^{\circ} \mathrm{C}$. Because of the inlet temperature of aeroengine is too high, in order to increase temperature bearing ability, hot components such as turbine vanes and blades of aeroengine have to upgrade their materials' temperature bearing ability and use thermal barrier coating and cooling hole techniques[1,2].

Cooling holes can greatly increase working temperature of aeroengine and are widely used in modern aero-engine. The cooling hole quantity of one modern aero-engine can reach to several ten thousands[3]. The typical dimensions of cooling holes are shown in Table1[4]. These cooling holes have characteristics such as great quantity, complicate spatial distribution, small volume and high requirement of machining quality. And only laser technique can be used to machine cooling holes on thermal barrier coating of aeroengine turbine blades, as thermal barrier coating is ceramic material whose conductivity is poor[5,6].

Traditional machining methods of cooling hole are laser(traditional laser) machining, electric discharge machining and electro-stream machining. These machining methods can induce defects 
such as thick recasting layer, crack, large heat-affected area or conductivity requirement of the machining material[1,7,8]. And these defects have caused several accidents during aeroengine tests and service. Therefore, new cooling hole machining technique is required.

Table1 Typical cooling hole dimensions for different aeroengine components

\begin{tabular}{|c|c|c|c|c|}
\hline Component & Diameter,mm & Wall thickness, $\mathrm{mm}$ & Angle to surface, deg & Number of holes \\
\hline Blade & $0.3-0.5$ & $1.0-3.0$ & 15 & $25-200$ \\
\hline Vane & $0.3-1.0$ & $1.0-4.0$ & 15 & $25-200$ \\
\hline Afterburner & 0.4 & $2.0-2.5$ & 90 & 40000 \\
\hline Baseplate & $0.5-0.7$ & 1.0 & $30-90$ & 10000 \\
\hline Seal ring & $0.95-1.05$ & 1.5 & 50 & 180 \\
\hline Cooling ring & $0.78-0.84$ & 4.0 & 79 & 4200 \\
\hline Cooling ring & 5.0 & 4.0 & 90 & 280 \\
\hline
\end{tabular}

Femtosecond laser has very short pulse duration,very high fluence. When femtosecond laser is used for material processing, the process exhibits characteristics such as multi-photon absorption and ionization threshold which are only controlled by materials' atom characteristics, prcecision threshold, small heat-affected areas. Therefore, femtosecond laser can be used for any material's micro-process, without limitation of material type and characteristics. Femtosecond laser processing technique can overcome defects induced by traditional processing techniques, is the main investigated subject of aeroengine cooling hole processing technique [9-11]

In this paper, we first introduce advantages and research state of femtosecond laser processing cooling holes in aeroengines. Subsequently, we discuss problems and challenges of femtosecond laser processing cooling holes in aeroengines. At last, we present the key research areas of femtosecond laser processing cooling holes in aeroengines.

\section{Femtosecond laser in micro-hole processing}

Due to the ultrashort pulse width which is shorter than the characteristic times of most physicochemical process(such as electronic relaxation time and electron-phonon relaxation time), regulation of electron states and "nonthermal process" can be realized during material processed by femtosecond laser. And high intensity and peak power density which can reach to $10^{22} \mathrm{~W} / \mathrm{cm}^{2}$ of femtosecond laser let fast material processing be true. Compared with material processing by long pulse laser, the interaction of femtosecond laser with free electron, ion, plasma and atom is a nonlinear and non-equilibrium procedure. Advantages of femtosecond laser in micro-hole processing are represented in the next paragraph[9-14].

(1) Recast layer is very thin or vanish during micro-hole processed by femtosecond laser.

The laser ablation process of metal can be explained by the course of excited electron changes from ionization to stabilization after material ablated in the time scale. Quasi thermal equilibrium state of electron appear in $10^{-13} \mathrm{~S}$ owing to scattering effect. At this point, temperature of the electron is much higher than that of its surrounding lattice. In $10^{-13} \mathrm{~S} \sim 10^{-12} \mathrm{~S}$, electrons with quasi thermal equilibrium state begin to radiate phonon which transfers energy to the crystal lattice and induces electron-phonon coupling. Energy distribution is close to the thermal equilibrium state, relaxation of electrons through $10^{-12} \mathrm{~S} \sim 10^{-11} \mathrm{~S}$. Then, heat begins to diffuse to the surrounding material through electron drift and coupling of crystal lattice. When the energy absorbed by the material reaches its melting point, the phase transition from solid state to liquid state occurs. Ablation procedure of visible laser on time scale can be divided into non melting process and melting process, and the heat balance time of $10^{-12} \mathrm{~S} \sim 10^{-11} \mathrm{~S}$ is their the dividing line. Because of the femtosecond laser pulse width is far less than the time of material just happened electron-phonon coupling, so cooling and heat diffusion process of electron temperature can be negligible in the duration of femtosecond laser. The laser energy absorption is restricted in a very small area, and electron temperature reaches very high in a very short period of time, the material changes from solid to plasma in the form of jet from the matrix. while, the surrounding material is still in the "cold" state. Which realizes non hot melt processing by femtosecond laser. So, this is the non melting processing of femtosecond laser. Compared with the long pulsed laser, the micro-holes machined by femtosecond laser are more regular and accurate. 
(2)Micro machining with small size

Due to restriction of the diffraction pattern, sizes of micro-holes processed by laser are generally larger than the laser wavelengths.

Although the focal spot size of femtosecond laser can not be less than half of its wavelength with diffraction limit, but because of high peak power, and material interaction for multiphoton process, which has the transverse distribution of Gauss laser and material interaction, if the focus center intensity reaches the multiphoton ionization threshold, processing range of the material can not be limited in the whole focal range, but limited in small range near the center of the laser focus which is far less than the focal spot. Therefore, the processing scale of femtosecond laser can up to $1 / 20$ wavelength, i.e. several tens nanometers.

(3)Wide application

Femtosecond laser has ultrashort pulse width, high power density, so that it can theoretically process any material. And femtosecond laser makes up for the shortcomings of common micro-hole processing methods. While electron beam machining and focused ion beam machining require harsh conditions. Electric spark erosion can only be used in the processing of conductive materials. Electrochemical drilling has very low efficiency and is limited by material property. And mechanical drilling can not be used in the process of very hard materials.

\section{Research state of the micro-hole machining of aero engine by femtosecond laser}

The mechanism of femtosecond laser ablation of metallic materials is complex. Processing methods and process parameters such as pulse width, integral flux, wavelength, pulse repetition frequency and the size of the laser beam will influence the processing effect.

According to the relative motion of laser and material, femtosecond laser drilling methods of metal materials can be divided into the following 4 types: single pulse, percussion, trepanning and helical drilling. Generally, laser percussion is considered to be ideal for thick-section workpiece drilling due to the low pulse energy requirement and insignificant heat damages. The common methods used in the micro-hole processing of aeroengines are percussion and trepanning.

The result of laser micromachining of a second generation single crystal superalloy with femtosecond pulses shows that :there are not conventional laser processing defects including spatter, recast layers, heat-affected zones and microcracks around the laser machined region at laser fluences in the range of $1 \sim 200$ times the ablation threshold; laser-induced plastic deformation produced dislocation substructures within a limited region to $2 \mathrm{um}$ around the processed region at a fluence close to the ablation threshold[15].

$\mathrm{Ma}[16]$ studied femtosecond laser ablation regimes in a single crystal superalloy, found that: the ablation rates of the superalloy exhibit two logarithmic dependences on the fluence of the laser pulse; in the low fluence regime, the ablation rate of the superalloy is determined by the optical absorption length. In the high fluence regime, the ablation rate of the superalloy increases dramatically and has a logarithmic dependence of the fluence. Semaltianos[17] investigated femtosecond laser ablation characteristics and mechanisms of the nickel based superalloy C263, found that : the debris of C263 oxidised crystalline nanoparticles. For low fluences, the central surface morphology of multipulse ablated craters(dimples) is characterized by ripples which exhibit the laser wavelength periodicity, while at high fluences melting and resolidification is observed.

Results of our study on micro-hole machining of DZ22 superalloy by femtosecond laser show that exit side shapes of hole significantly changes from oval to irregular shape and maicro-cracks are found in the wall of hole in some processing parameters, as shown in Fig.1 and Fig. 2 . 


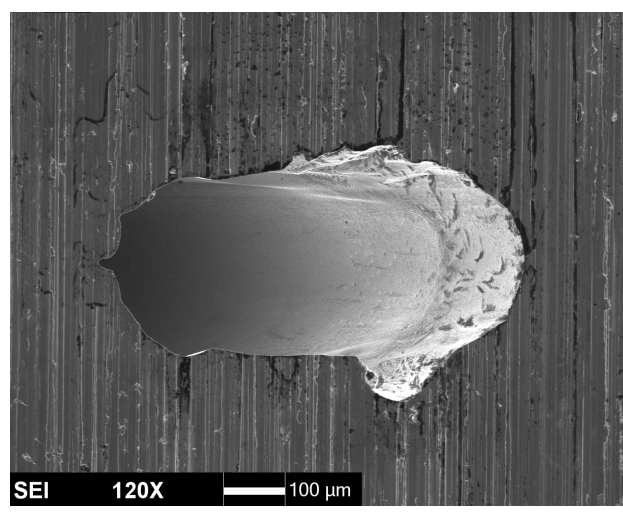

Figure 1. Exit side shape of the hole

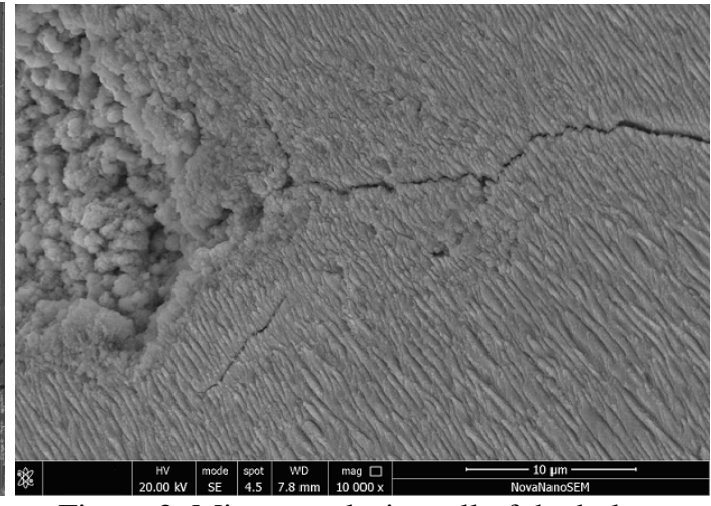

Figure 2. Micro-cracks in wall of the hole

Investigation of femtosecond laser machining of single-crystal superalloys through both plasma-sprayed and EB-PVB thermal barrier coatings(TBC) shows that: a complete absence of conventional laser processing defects including spatter, recast layers, heat-affected zones and micro-cracks around the laser-machined region through the $\mathrm{TBC}$, bond coat and superalloy substrate and no laser-induced delamination along interfaces of the TBC systems occurred due to the minimization of heat conduction[18].Study of femtosecond laser machined cooling holes generated on thermal barrier coated CMSX4 superalloy by Das[19] shows that: the threshold fluence was not appreciably dependent on the initial roughness of the coating surface, circular holes machined on thermal barrier coated CMSX4 superalloy samples have a good surface finish with a Ra value of about 1.8um, and the process did not cause any severe collateral damage such as the recast layer and micro-cracking and delamination of the 7YSZ layer during machining or during thermal cycling of the coating at $1100^{\circ} \mathrm{C}$, but have a certain degree of taper due to the Gaussian energy distribution of the laser pulses used and minor ablative material lossed from the surface of the coating around the holes occurred during machining[20]。

\section{Existing problems and research focus}

There are still some problems in the micro-hole processing of aeroengine components by femtosecond laser, such as poor hole shape, recast layer, micro-crack, large surface roughness of the hole wall and delamination of thermal barrier coating. Most research on the micro-hole processed by femtosecond laser of aeroengine is still in the laboratory stage and mainly focus in the micro-hole drilling of aeroengine materials.

The main reasons for the above problems are:(1) The damage mechanism of materials during femtosecond laser processing is unclear, and the model can not be established to describe the whole process of micro-hole machining. (2) Due to the nonlinear effect and ultrafast characteristics of femtosecond laser processing, it is difficult to couple these conditions, and this leads to the research of femtosecond laser processing technology is not deep enough. The basic and regular research on the technology of micro-hole machining by femtosecond laser is needed, especially research on the relationship of the influencing factors.

On the other hand, the thermal barrier coating is a complex solid system with characteristics such as porous, multi interface, multi crystal, the failure modes are greatly influenced by microstructure and physical properties of ceramic layer, formation of TGO which induced increase of the interface amount[21].Femtosecond laser processing of cooling holes first destroy the integrity of TBC, followed by a variety of defects appeared in the process of making hole. This may cause the failure behavior of thermal barrier coatings changes (unlike failure behavior of thermal barrier coating with complete surface) and promote the early failure during service.

The mechanism of interaction between femtosecond laser and metal material has not yet fully understood, especially the research of interaction between femtosecond laser and high temperature alloys used in aeroengine is very lack, compared to the research of femtosecond laser processing technology. 
The key points of research on micro-hole drilling of the aeroengine by femtosecond laser are comprehensive explanation of the damage mechanism of aeroengine material processed by femtosecond laser, improvement of multiscale models which describe the whole process of micro-hole machining, grasp of influence mechanism and law of the processing parameters on the micro-hole machining process, and establishment of method for regulating the process parameters of micro-hole machining by femtosecond laser.

\section{Conclusions}

Femtosecond laser has the characteristics of ultrashaort pulse width and high power density, can be used for any material micro-process, without limitation of material type and characteristics. Femtosecond laser processing technology can make up for a series of defects in the traditional processing of aeroengine cooling holes, and is an important research content in the field of cooling hole processing.

There are still some problems in the micro-hole processing of aeroengine components by femtosecond laser, such as poor hole shape, recast layer, micro-crack, large surface roughness of the hole wall and delamination of thermal barrier coating.

The key points of research on micro-hole drilling of aeroengine by femtosecond laser are comprehensive explanation of the damage mechanism, improvement of multiscale models which describe the whole process of micro-hole machining, grasp of influence mechanism and law of the processing parameters on the micro-hole machining process, and establishment of method for regulating the process parameters of micro-hole machining by femtosecond laser.

\section{Acknowledgements}

This work was financially supported by the Aero-science Foundation (2014ZE21009).

\section{References}

[1] H.N. Zhu, X.X.Qi. Development of machining technology gas holes on turbine blades. Aeronautical manufacturing technology. 13(2011),P.71-74.

[2] H.Y. Qi,Y.M.Wang. andX.G.Yang.Thermal fatigue of atmospheric plasma sprayed thermal barrier coating. Failure analysis and prevention. 3 (1) (2008) ,P.15-18.

[3] A. Corcoran, L. Sexton.\& B. Seaman. The laser drilling of multi-layer aerospace material systems. Journal of Materials Processing Technology.23(1) (2002) ,P. 100-106.

[4] M. H. H. Van Dijk, J. E. De Vlieger and J. E. Brouwer. Sixth Int.Conf. on 'Lasers in manufacturing', Birmingham, UK, IFS Publications, 23(1989) ,P.7 - 247.

[5] J. Girardot, M. Schneider and L.Berthe. Investigation of delamination mechanisms during a laser drilling on a cobalt-base superalloy. Journal of Materials Processing Technology. 213(2013) ,P. 1682-1691.

[6] R.F. Sun,X.B.Zhang and W.B.Cao. Laser Drilling of Ni-Base Single-Crystal Superalloy through Thermal Barrier Coatings.Rare metal materials and engineering.43(5) (2014) ,P.1193-1198.

[7] J .Liu.. Drilling technique of aeroengine cooling hole.Aeroengine. 2 (1995) ,P. 31-36.

[8] W.Y. Guo,M.C.wang and X.B.Zhang. Recast layer formed by laser drilling of Ni- based superalloys and progress on its control. 24 (4) (2003),P.1-3.

[9] C. Tan. Research on processing technology and surface quality of metal micro-holes machined by femtosecond laser.Changsha,Zhongnan University. ( 2014),P.1-17. 
[10] H.N.Jia. Technique research of femtosecond laser pulses for drilling the shaped micro-hole of turbine blades.Beijing, University of Chinese Academy of Sciences. (2013) ,P.1-26

[11] F. He, Y.Cheng. Femtosecond Laser Micromachining: Frontier in Laser Precision Micromachining. 34(5) (2007) ,P.595-622.

[12] D. Von der Linde, T.K. Sokolowski and J. Bialkowski. Laser-solid interaction in the femtosecond re gime[J]. Applied Surface Science.109(1997) ,P.1-10.

[13] B. Rethfeld, T. K.Sokolowski and D. Von der Linde. Timescales in the response of materials to femtosecond laser excitation[J]. Applied Physics A , 79(46) (2004) ,P.767-769.

[14] B. Xia,L.Jiang and S.M. Wang. Femtosecond laser drilling of microholes. China journal of lasers, 40(2) (2013) ,P. 1-12.

[15] Q. Feng, Y.N. Picard and H. Liu. Femtosecond laser micromachining of a single-crystal superalloy [J]. Scripta Materialia, 53(5) ( 2005) ,P. 511-516.

[16] S. Ma, J.P.Mcdonald, B.Tryon and M.YalisoveFeemtosecond laser ablation regimes in a single crystal superalloy.Metallurgical and materials transactions A,Vol.38, (2007) ,P. 2349-2357.

[17] N.G. Semaltianos, W. Perrie and P. French. Femtosecond laser ablation characteristics of nickel-based superalloy C263[J].Applied ZHUPhysics A Material Science \& Processing.94(2009),P.999-1009

[18] Q. Feng, Y.N. Picard and J.P. McDonald. Femtosecond laser machining of single-crystal superalloys through thermal barrier coatings[J]. Materials Science and Engineering A, 430(2006) ,P. 203-207.

[19] D. K. Das, J.P.McDonald and S.M.Yalisove. Femtosecond pulsed laser damage characteristics of 7\% Y2O3-ZrO2 thermal barrier coating. Applied Physics A Material Science \& Processing, 91( 2008), ,P.421-428.

[20]D. K Das, M. P. Tresa. Femtosecond laser machining of cooling holes in thermal barrier coated CMSX4 superalloy[J]. Journal of Materials Processing Technology. 209(2009) ,P.5661-5668.

[21] H.B. Xu, S.K.Gong and L.Q.Chen. Failure process of thermal barrier coatings under thermal and mechanical loading. Journal of Beijing University of Aeronautics and Astronautics. 30(10) ( 2004),P.919-924. 